\title{
Scaling in the two-dimensional U(1)-Higgs model
}

\author{
Hermann Dilger and Jochen Heitger* \\ Institut für Theor. Physik I, Universität Münster, Wilhelm-Klemm-Str. 9, D-48149 Münster, Germany
}

We study the continuum limit of the $2 D \mathrm{U}(1)$-Higgs model with variable scalar field length, which is qualitatively different from the fixed length case. Our simulations concentrate on the scaling behaviour of the topological susceptibility, and an instanton-induced confinement mechanism of fractional charges is numerically confirmed.

\section{Introduction}

The $2 D$ abelian Higgs model shares prominent features of the SU(2)-Higgs sector of the Standard Model related to baryon number violation. Whereas detailed studies of the model with various methods are available [1,24, it is not well understood within the euclidean lattice approach, above all for variable scalar field length. We examine the continuum limit in this case and, in particular, investigate the scaling behaviour of the topological susceptibility.

\section{Simulation of the lattice model}

On a two-dimensional lattice $\Lambda$ (with spacing $a$, extensions $L_{\mu}$, and unit vectors $\left.\hat{\mu}, \mu=1,2\right)$ the action is given by $S=S_{\mathrm{g}}+S_{\phi}$,

$$
\begin{aligned}
S_{\mathrm{g}}= & \beta \sum_{x \in \Lambda}\left(1-\operatorname{Re} U_{\mathrm{p} ; x}\right) \\
S_{\phi}= & \sum_{x \in \Lambda}\left\{-2 \kappa \sum_{\mu=1}^{2} \operatorname{Re}\left(\varphi_{x+\hat{\mu}}^{*} U_{x, \mu} \varphi_{x}\right)\right. \\
& \left.+\left|\varphi_{x}\right|^{2}+\lambda\left(\left|\varphi_{x}\right|^{2}-1\right)^{2}\right\}
\end{aligned}
$$

$U_{\mathrm{p} ; x} \equiv \mathrm{e}^{i F_{x}}$ is the Wilson plaquette. The gauge fields $A_{x, \mu}$ enter as phases of the links $U_{x, \mu}$, and the scalar field $\varphi_{x}$ is decomposed as $\varphi_{x}=\rho_{x} \mathrm{e}^{i \omega_{x}}$.

In the Monte Carlo simulation of this model a combination of metropolis and overrelaxation algorithms (for the $\varphi$-field as proposed in [3]) is applied. Since these local algorithms generally do not manage to tunnel between different topological sectors, we use so-called instanton hits 断.

\footnotetext{
*Presenter at the conference LATTICE 96, St. Louis.
}

These are updates in the gauge sector by a global proposal of an instanton configuration

$A_{x, \mu} \rightarrow A_{x, \mu} \pm \Delta A_{x, \mu}$

with $\Delta A_{x, \mu}$ carrying unit topological charge and being non-zero in a region of the instanton size.

We consider expectation values built up from the operators $\rho_{x}$ (scalar length), the $\varphi$-links

$L_{\varphi ; x \mu}^{ \pm} \equiv\left\{\begin{array}{l}\operatorname{Re}\left(\varphi_{x+\hat{\mu}}^{*} U_{x, \mu} \varphi_{x}\right) \\ \operatorname{Im}\left(\varphi_{x+\hat{\mu}}^{*} U_{x, \mu} \varphi_{x}\right)\end{array}\right.$,

and Wilson loops $W(R, T)$ of space-time extensions $R, T$. Particle masses in the Higgs $\left(m_{H}\right)$ and vector $\left(m_{W}\right)$ channels are extracted from fits of $\rho_{x}^{2}, L_{\varphi ; x 1}^{+}$and $F_{x}, L_{\varphi ; x 1}^{-}$-correlation functions, respectively.

\section{Lines of constant physics}

Let us mention some limiting cases of the model. For $\kappa=0$ one arrives at pure gauge theory (PGT) with confinement in two dimensions. $\lambda=\infty$ (fixed length case $\left|\varphi_{x}\right| \equiv 1$ ) and $\beta=\infty$ is the $2 D \mathrm{XY}$-model with its Kosterlitz-Thouless phase transition between a massive vortex phase $\left(\kappa<\kappa_{c}\right)$ and a massless spin wave phase $\left(\kappa>\kappa_{c}\right)$. At finite $\beta$ this transition is expected to become a crossover [1]. For any fixed $\lambda$ and $\beta \rightarrow \infty$ the vector mass $a m_{W}$ tends to zero, defining a continuum limit $(a \rightarrow 0)$, but $a m_{H}$ stays finite. Ending up with infinite $m_{H}$ at $\beta=\infty$ for all (fixed) $\lambda$ values reflects the freezing of the radial mode on large scales in the $2 D \phi_{n=2}^{4}$-theory [5].

Figure 1 illustrates the typical dependence of the Higgs and vector masses on $\kappa$. We find a change in the behaviour of the mass spectrum in addition to a rapid breakdown of the topological 


\begin{tabular}{ccccccccc}
\hline LCP & set & $\kappa$ & $a m_{H}$ & $a m_{W}$ & $R_{H W}$ & $v_{R}$ & $\chi_{\text {top }} \cdot 10^{4}$ & $\chi_{\text {top }} / m_{H}^{2} \cdot 10^{4}$ \\
\hline \hline & A & 0.2937 & $0.882(4)$ & $0.540(8)$ & $1.63(3)$ & $1.8736(1)$ & $0.105(4)$ & $0.14(1)$ \\
L1 & B & 0.2607 & $0.426(4)$ & $0.258(5)$ & $1.65(4)$ & $1.8751(1)$ & $0.030(2)$ & $0.17(1)$ \\
& C & 0.253 & $0.221(1)$ & $0.132(2)$ & $1.68(3)$ & $1.8772(1)$ & $0.011(1)$ & $0.21(2)$ \\
\hline \multirow{3}{*}{ L2 } & A & 0.2858 & $0.692(11)$ & $0.477(9)$ & $1.45(4)$ & $1.6144(2)$ & $1.62(2)$ & $3.4(2)$ \\
& B & 0.25885 & $0.330(5)$ & $0.228(5)$ & $1.45(4)$ & $1.6170(2)$ & $0.53(1)$ & $4.9(2)$ \\
& C & 0.2525 & $0.165(2)$ & $0.112(3)$ & $1.47(4)$ & $1.6146(5)$ & $0.181(4)$ & $6.7(3)$ \\
\hline \multirow{2}{*}{ L3 } & A & 0.2731 & $0.446(11)$ & $0.514(8)$ & $0.87(2)$ & $1.2739(5)$ & $14.8(1)$ & $75(4)$ \\
& B & 0.257 & $0.234(8)$ & $0.268(7)$ & $0.88(4)$ & $1.2757(5)$ & $4.12(3)$ & $75(6)$ \\
& C & 0.252 & $0.121(2)$ & $0.141(3)$ & $0.85(3)$ & $1.2746(5)$ & $1.21(1)$ & $83(3)$ \\
\hline
\end{tabular}

Table 1. LCP-parameters A: $\{\Lambda=16 \times 16, \beta=10, \lambda=0.01 / 0.013\}, B:\{\Lambda=32 \times 32, \beta=40, \lambda=0.0025\}$ and $C:\{\Lambda=64 \times 64, \beta=160, \lambda=0.000625\}$. All mass errors come from a jackknife analysis.
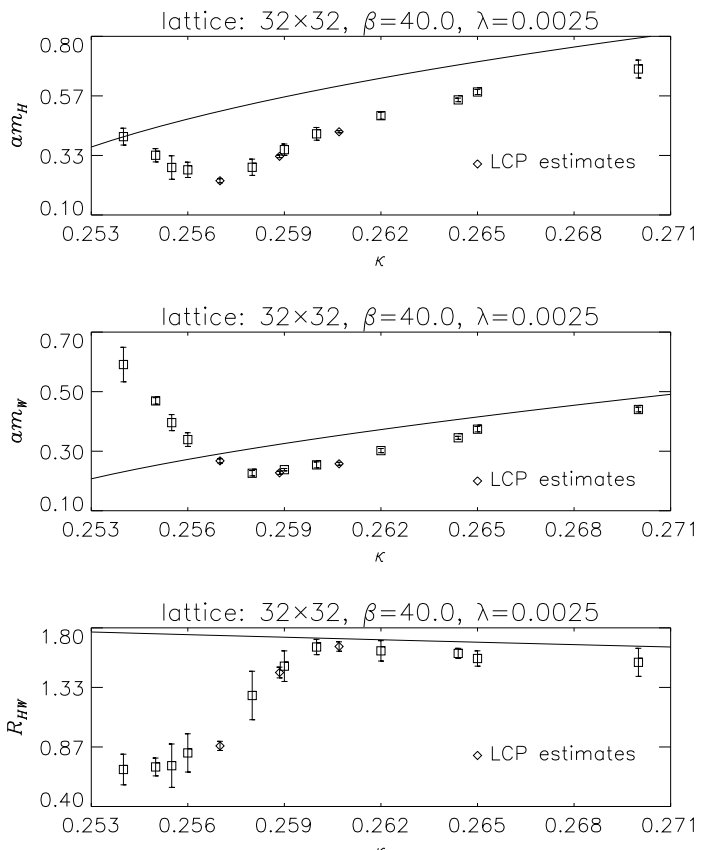

Figure 1. Higgs and vector $\left(L_{\varphi}^{-}\right)$masses for fixed $\beta$ and $\lambda$ and their classical relations (solid lines).

susceptibility around the crossover $\kappa$-value $\bar{\kappa}$ defined at minimal $a m_{W}\left(L_{\varphi}^{-}\right)$. The $a m_{W}$-estimates from $\varphi$-link $\left(L_{\varphi}^{-}\right)$and plaquette $(F)$ correlations are only consistent for $\kappa>\bar{\kappa}$. For decreasing $\kappa<\bar{\kappa}$ the $F$-correlations weaken strongly and show a decreasing mass. This corresponds to a massless photon in the PGT limit, which is, however, no physical degree of freedom. The qualitatively different behaviour of the $L_{\varphi}^{-}$-correlations in figure 1 is another striking analogy to the $4 D$ model [6], besides the fact that the $\kappa$-dependence of $a m_{H}$ and $a m_{W}$ is similar.

Now we set up the lines of constant physics (LCPs) by the requirements $a m_{H}, a m_{W} \rightarrow 0$ at fixed scalar field VEV and mass ratio:

$v_{R} \equiv \sqrt{2 \kappa}\langle\rho\rangle=\bar{v} \quad R_{H W} \equiv \frac{m_{H}}{m_{W}}=\bar{R}$.

With a tuning of $\kappa$ this can be achieved by $\beta \rightarrow \infty, \lambda \rightarrow 0$, realized for large enough $\beta$ by

$\beta \rightarrow \infty \quad \beta \lambda=$ constant.

The simulated points in parameter space are collected in table 11. One has $\beta \lambda \simeq 0.1$, and $\kappa$ was adjusted until the renormalization conditions (5) were simultaneously fulfilled within errors.

It has to be emphasized that the continuum limit (6), which amounts to send $\kappa \rightarrow \frac{1}{4}$ at the same time, see figure 2, should not be confused with the gaussian limit. The crucial point is that the relation between the dimensionful bare continuum couplings $\lambda_{0}, e_{0}$ and the lattice parameters is $\lambda \propto a^{2} \lambda_{0}$ and $\beta=1 / a^{2} e_{0}^{2}$. Hence $\lambda \rightarrow 0$ at constant $\beta \lambda$ does not imply $\lambda_{0} \rightarrow 0$ for $a \rightarrow 0$.

\section{Topological susceptibility}

We adopt the geometric definition of the topological charge, which in two dimensions reads

$Q_{\text {top }} \equiv \frac{e_{0}}{4 \pi} \int \mathrm{d}^{2} x \epsilon_{\mu \nu} F_{\mu \nu}(x) \longrightarrow \frac{1}{2 \pi} \sum_{x \in \Lambda} F_{x}$

and has only integer values. The topological susceptibility is $\chi_{\text {top }} \equiv \frac{1}{\Omega}\left\langle Q_{\text {top }}^{2}\right\rangle, \Omega$ : lattice volume, 

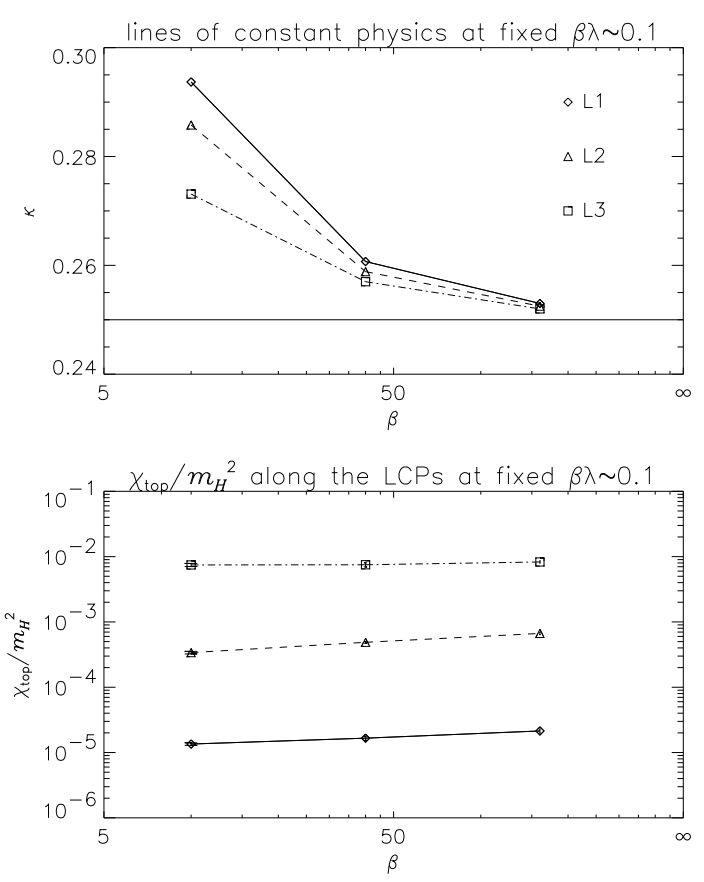

Figure 2. Scaling of $\chi_{\text {top }}$ along the LCPs.

and has been measured on the LCPs leading to the results in table 1 and figure 2. Significant finite volume effects are ruled out. Within the chosen parameter sets $\chi_{\text {top }}$ varies by orders of magnitude, and a contraction of the $\kappa$-region, which is limited by a still measurable $\chi_{\text {top }}$ from above and by the line L3 from below, is seen. Note that this LCP already lies close to PGT, where the $\beta$-dependence $\chi_{\text {top }} \rightarrow \frac{1}{4 \pi^{2} \beta}$ for $\beta \rightarrow \infty, \Omega \rightarrow \infty$ is known. Except for L3, the scaling of the dimensionless ratio $\chi_{\mathrm{top}} / m_{H}^{2}$ is rather poor.

Finally we look for confinement by instantons, suggested for this model in 77. Using $\oint_{\partial \mathcal{A}} A_{\mu} \mathrm{d} x_{\mu}=\int_{\mathcal{A}} \mathrm{d}^{2} x F_{12}$ we obtain a unique lattice prescription for the Wilson loop with fractional test charge $q$ in the compact formulation:

$$
W_{q}(R, T)=\mathrm{e}^{i q \sum_{x \in \mathcal{A}_{R, T}} F_{x}} \mathcal{A}_{R, T} \in \Lambda \text { : area. }
$$

Since $F_{x}=e_{0} a^{2} F_{12}(x)$ for $a \rightarrow 0$, one requires $F_{x} \in[-\pi, \pi)$, so $2 \pi$-ambiguities for $q=\frac{1}{2}$ as for the standard form with $A_{x, \mu}$ are avoided. The static potential $V_{q}=-\lim _{T \rightarrow \infty} \frac{1}{T} \ln W_{q}$ gets in the dilute instanton gas approximation a contribution $\chi_{\text {top }}\left\{1-\cos \left(2 \pi q / e_{0}\right)\right\} R$, which signals con- finement for non-integer $q / e_{0}$. We take Polyakov loop correlations $\left.P_{q}(R) \equiv W_{q}(R, T)\right|_{T=L_{2}}$ and fit $V_{q}=-\frac{1}{q^{2} L_{2}} \ln P_{q}$ to a continuum Yukawa ansatz

$V_{q}(R)=\frac{e_{R}^{2}}{2 m_{s}}\left(1-\mathrm{e}^{-m_{s} R}\right)+\alpha R$.

As exemplarily displayed in table 2 for $q=\frac{1}{2}$ in L2, lying just in the Higgs regime $(\kappa>\bar{\kappa})$, the meaning of the fit parameters $a e_{R}$ (renormalized gauge coupling, small corrections to $a e_{0}=1 / \sqrt{\beta}$ expected), $a m_{s}$ (screening mass, $\left.\simeq a m_{W}\right)$ and $\alpha$ $\left(=2 \chi_{\mathrm{top}} / q^{2}\right)$ is reproduced.

\begin{tabular}{cccc}
\hline set & $a m_{s}$ & $a e_{R}$ & $\alpha / 8 \cdot 10^{4}$ \\
\hline \hline $\mathrm{A}$ & $0.436(2)$ & $0.3136(3)$ & $1.5(1)$ \\
$\mathrm{B}$ & $0.209(2)$ & $0.1551(3)$ & $0.55(6)$ \\
$\mathrm{C}$ & $0.098(3)$ & $0.0769(5)$ & $0.20(5)$ \\
\hline
\end{tabular}

Table 2. Fit parameters of $V_{\frac{1}{2}}$ in $L 2$.

\section{Discussion and outlook}

The continuum limit in the $2 D \mathrm{U}(1)$-Higgs model with variable scalar field length seems to be achieved as outlined in (6). The scaling of $\chi_{\text {top }}$ is still unclear and will be studied further. Also the systematic errors by the statistical uncertainties in the conditions (5) should be estimated. The LCPs give strong evidence for a phase transition in $\kappa=\frac{1}{4}$ at $\beta=\infty$ and for a crossover for $\beta<\infty$.

\section{REFERENCES}

1. D. Jones, J. Kogut, D. Sinclair, Phys. Rev. D19 (1979) 1882; I. Ichinose, H. Mukaida, Int. J. Mod. Phys. A9 (1994) 1043.

2. S. Grunewald, E.-M. Ilgenfritz, M. MüllerPreussker, Z. Phys. C33 (1987) 561.

3. B. Bunk, Nucl. Phys. B42 (Proc. Suppl.) (1995) 566.

4. F. Fucito, S. Solomon, Phys. Lett. B134 (1984) 230; H. Dilger, Int. J. Mod. Phys. $\underline{\text { C6 }}$ (1995) 123.

5. K. Pinn, Z. Phys. $\underline{\text { C45 }}$ (1990) 453; private communication.

6. H. G. Evertz et al., Nucl. Phys. B285 [FS19] (1987) 559 and 590.

7. C. Callen, R. Dashen, D. Gross, Phys. Lett. $\underline{B 66}$ (1977) 375. 